\title{
PROPIEDADES PSICOMÉTRICAS DEL CUESTIONARIO DE AFRONTA- MIENTO AL ESTRÉS PARA PACIENTES ONCOLÓGICOS (CAEPO)
}

\section{PSYCHOMETRIC PROPERTIES OF THE COPING TO STRESS QUESTIONNAIRE FOR ONCOLOGIC PATIENTS}

\author{
Ana García-Conde', Melina Miaja Avila², Rocío Romero Retes', Elena Ibáñez Guerra ${ }^{3}$ y \\ José Soriano Pastor ${ }^{3}$ \\ l Fundación Instituto Valenciano de Oncología, Valencia, España \\ 2 Facultad de Psicología, Universidad Autónoma de Nuevo León. Monterrey, NL, México. \\ ${ }^{3}$ Facultad de Psicología, Universidad de Valencia, Valencia, España.
}

Resumen

Los objetivos del estudio fueron: 1) analizar la estructura factorial de la escala CAEPO mediante análisis factorial exploratorio y confirmatorio; 2) estimar la consistencia interna de los factores; 3) describir la distribución de los factores; 4) analizar las diferencias de medias de las estrategias de afrontamiento entre hombres y mujeres. El Cuestionario de Afrontamiento al Estrés para Pacientes Oncológicos (CAEPO) de González (2004) fue aplicado a 148 pacientes oncológicos. Se sugiere reducir la escala a 35 ítems. Se encontró una estructura de cuatro factores correlacionados con consistencias internas de adecuadas a altas. La distribución de los factores se ajustó a una curva normal y no se encontraron diferencias significativas entre hombre y mujeres. En futuras investigaciones, se sugiere su empleo en muestras clínicas de España y otros países hispanoparlantes.

Palabras clave: Afrontamiento activo, rumiación, pasividad, evitación, cáncer.

\section{Abstract}

The goals of the study are: 1) to analyze the factorial structure of the Coping to Stress Questionnaire for Oncologic Patients instrument by the use of a factorial exploratory and confirmatory analysis; 2) to estímate the internal reliability of the factors; 3 ) to describe the distribution of the factors; 4) to analyze the mean differences of the coping strategies between men and women. The Coping to Stress Questionnaire for Oncologic Patients (González, 2004) was used for 148 oncologic patients. It is given suggestions to reduce the questionnaire to 35 items, It was found four correlatonated factors estructure with internal reliability from adecuated to high. The factors distribution was adjusted to the normal curve and it was not found significative differences between men and women. In future investigation, it is suggested to use this questionnaire un clinic samples from Spain and othes spanishspeaking countries.

Keywords: Active coping, ruminate, passiveness, avoidance, cancer.

\section{Correspondencia:}




\section{INTRODUCCIÓN}

Las personas que se enfrentan a un proceso de enfermedad crónico-degenerativo experimentan un gran número de estresores, cambios y pérdidas ${ }^{(1,2)}$. En pacientes con cáncer los estresores y cambios más relevantes son: la pérdida de la salud, los cambios en la apariencia física como la mastectomía, la pérdida de cabello o peso corporal $^{(3)}$, pérdida de la función sexual(4), la pérdida del ideal del futuro $^{(5)}$, las restricciones en actividades físicas, la pérdida de la rutina diaria como la asistencia al trabajo/clases o la convivencia con la familia debido a las constantes visitas médicas u hospitalizaciones, la imposición de un régimen alimentario que en muchas ocasiones no gusta al paciente y el seguimiento de un tratamiento que suele provocar sensaciones dolorosas, a veces más incómodas que la propia enfermedad $^{(6,5)}$.

Todo lo anterior, hace factible que se dificulte la adaptación a la enfermedad y que el cáncer sea considerado como una experiencia estresante, difícil y traumática. No obstante, el estrés no sólo se debe al entorno, ni es consecuencia exclusiva de características de personalidad, sino que más bien es consecuencia de la valoración subjetiva del estresor, de la capacidad del individuo para controlar la situación estresante, y de la influencia de los patrones de conducta aprobados por la sociedad ${ }^{(7,8)}$.

La manera de afrontar el proceso de enfermedad repercute en la calidad de vida de la persona, en su sistema inmunológico, y en consecuencia en el proceso del cáncer o la aceleración del desarrollo del mismo. En este sentido, Mera y Ortiz ${ }^{(9)}$ hallaron en mujeres con cáncer de mama que la calidad de vida era predicha por mayor apoyo social como estrategia de afrontamiento activa y menor autocrítica como estrategia de afrontamiento pasiva. Un mejor ajuste al cáncer de mama esta asociado con menor uso de estrategias de afrontamiento pasivas como la evitación y negación y con mayor uso de estrategias de afrontamiento centradas en el problema como la aceptación, reconceptualización positiva y búsqueda de apoyo social ${ }^{(10)}$. Además, Cardenal, et al. ${ }^{(11)}$ encontraron que altas puntuaciones en ira internalizada y en las estrategias de afrontamiento de resignación, autocrítica y baja puntuación de concienciación eran factores de riesgo que predecían una desfavorable evolución del cáncer.

El concepto de afrontamiento fue definido por Lazarus y Folkman ${ }^{(12)}$ como los esfuerzos cognoscitivos y/o conductuales que se desarrollan para manejar las demandas específicas externas o internas generadoras de estrés que valoraba el individuo como desbordantes de sus recursos. Por lo tanto, el afrontamiento es un proceso en el que constantemente se realizan esfuerzos para afrontar la situación que se percibe como amenazante o excedente de lo que el individuo puede hacer.

Existen múltiples instrumentos para medir el afrontamiento de forma general: como el de Lazarus y Folkman ${ }^{(12)}$, el de Sandín y Chorot $^{(13)}$, y el Moos ${ }^{(14)}$. Sin embargo, cuando hablamos de instrumentos específicos de evaluación del afrontamiento aplicados al cáncer el número se reduce considerablemente, siendo los más utilizados el MAC de Watson et al. ${ }^{(15)}$ y Mini MAC de Watson et al. ${ }^{(16)}$.

Uno de los instrumentos más actualizados y utilizados en el ámbito hispanohablante es el desarrollado por González ${ }^{(1)}$, el cual ha sido desarrollado específicamente para pacientes oncológicos. El Cuestionario de Afrontamiento al Estrés para Pacientes Oncológicos (CAEPO) fue desarrollado en población española. El CAEPO permite medir las estrategias de afrontamiento (cognitivas y conductuales) que utilizan los pacientes oncológicos al enfrentarse al proceso de enfermedad. 
EI CAEPO está integrado por siete factores de primer orden: Enfrentamiento y Lucha Activa (ELA): valoración de la situación como un reto o prueba, adoptando así un papel activo ante la enfermedad. Autocontrol y de Control Emocional (ACE): disminución o eliminación de los estados afectivos displacenteros que le produce la enfermedad. Búsqueda de Apoyo Social (BAS): búsqueda de apoyo afectivo, físico y moral de las personas cercanas a él. Ansiedad y Preocupación Ansiosa (APA): valoración de la situación como grave y amenazante, manifestando incertidumbre y ansiedad respecto al futuro, pronóstico y evolución de la enfermedad. Pasividad y Resignación Pasiva (PRP): valoración de la situación con escasas posibilidades para superarla, destacando una visión pesimista y fatalista respecto al futuro, evolución y pronóstico de la enfermedad y adoptando un papel pasivo ante la enfermedad. Escala de huida y Distanciamiento (HD): valoración de la situación como inadecua$\mathrm{da}$, intentando escapar o eludir la realidad de la situación. Escala de Negación (N): se refiere a que la persona niega la evidencia de la enfermedad y la realidad.

A partir de los sietes factores de primer orden, González ${ }^{(1)}$ define dos factores de segundo orden: Afrontamiento positivo conformado por la suma de los tres factores que representan conductas y pensamientos activos y positivos ante la situación de enfermedad (ELA, ACE y BAS) y Afrontamiento negativo conformado por la suma de los cuatro factores que representan conductas y pensamientos pasivos y negativos ante la situación de enfermedad (APA, PRP, HD y N).

Actualmente se está realizando mucha investigación sobre las estrategias de afrontamiento que utilizan los pacientes con cáncer para hacer frente al proceso de enfermedad $^{(17-19)}$. Aunque el CAEPO se muestra como un buen instrumento para medir el afrontamiento en población oncológica ${ }^{(1,19)}$, sus propiedades psicométricas han sido poco estudiadas presentando problemas de consistencia interna y estructura factorial en diversos estudios con muestras clínicas ${ }^{(20-22)}$.

Utilizando una muestra española de pacientes con cáncer García-Conde (21) observó que únicamente las estrategias de ELA, ACE y APA poseían valores de consistencia interna altos (de 0,74 a 0,82), mientras que las restantes estrategias de afrontamiento (BAS, PRP, HD y N) mostraron valores de consistencia interna bajos (de 0,21 a 0,57).

En otro estudio realizado en pacientes oncológicos peruanos, Díaz y Yaringaño (20) hallaron valores de consistencia interna opuestos a los encontrados por GarcíaConde $^{(21)}$. Las estrategias de primer orden BAS, PRP, HD y $\mathrm{N}$ mostraron valores de consistencia interna adecuados (de 0,62 a $0,68)$, así como el factor de segundo orden de afrontamiento positivo $(\alpha=0,64)$. Las estrategias de ELA, ACE y APA mostraron valores de consistencia interna bajos (de 0,43 a 0,59). Únicamente el factor de segundo orden de afrontamiento negativo tuvo consistencia interna alta $(\alpha=0,71)$.

Por su parte, Orive, et al. ${ }^{(22)}$ aplicaron el CAEPO en una muestra de pacientes españoles con tres enfermedades crónicas diferentes (hepatitis C crónica, enfermedad inflamatoria intestinal y vértigo recurrente). Los valores de consistencia interna que obtuvieron fueron altos para ELA, PRP, ACE y APA (de 0,71 a 0,78), adecuado para BAS (0.61), y bajos para HD y $N(0,41$ y 0,23 , respectivamente). Los autores al no encontrar la estructura factorial original de siete factores por el análisis factorial exploratorio, propusieron una estructura de cuatro factores: el primer factor definido por enfrentamiento y lucha activa, y autocontrol y de control emocional; el segundo factor por ansiedad y preocupación ansiosa; el tercer factor por pasividad $y$ resignación pasiva; y el cuarto factor por búsqueda de apoyo social. 
El estudio aquí presentado tiene como objetivos: 1) analizar la estructura factorial de la escala utilizando técnicas de análisis factorial exploratorio y confirmatorio; 2) estimar la consistencia interna de los factores; 3) describir la distribución de los factores; 4) analizar las diferencias de medias de las estrategias de afrontamiento entre hombres y mujeres.

Como resultados se espera obtener: 1) una estructura de siete factores independientes $^{(1)}$; 2) valores de consistencia interna altos $(\alpha \geq 0,70)$ para los factores de enfrentamiento y lucha activa, autocontrol y control emocional, y ansiedad y preocupación ansiosa ${ }^{(21,22)}$ y valores de consistencia interna bajos para los factores de huida y distanciamiento, y negación ${ }^{(20-22)}$; 3) distribuciones asimétricas negativas para los factores de enfrentamiento y lucha activa y autocontrol y control emocional y distribuciones asimétricas positivas hacia valores de baja negación ante la enfermedad (1); 4) mayor presencia de búsqueda de apoyo social en mujeres y de autocontrol y control emocional en hombres negación ${ }^{(23,24)}$.

\section{MÉTODO}

\section{Participantes}

Los criterios de inclusión fueron: tener de 18 años en adelante, tener una enfermedad oncológica diagnosticada, ser paciente de la Fundación Instituto Valenciano de Oncología (IVO), saber leer y escribir para comprender adecuadamente las instrucciones. Y como criterios de exclusión: incomprensión del idioma castellano, tener alteración cognitiva, padecer enfermedad psiquiátrica grave y tener fatiga excesiva que dificultase la atención.

La muestra quedó formada por 122 $(82,4 \%)$ mujeres y $26(17,6 \%)$ hombres. La media de edad fue de 52,47 años $(\mathrm{DT}=12,73)$. El 23,6\% $(\mathrm{n}=35)$ tenía estudios primarios, $25 \%(n=37)$ de enseñanza obligatoria, $27 \% \quad(\mathrm{n}=40)$ formación profesional y el $24,3 \% \quad(n=36)$ estudios universitarios. El 45,9\% $(n=68)$ de los casos fueron cáncer de mama, 21,6\% $\quad(n=32)$ cáncer ginecológico, $13,5 \% \quad(n=20)$ cáncer urológico, 3,4\% $(n=5)$ cáncer de pulmón, 3,4\% $(\mathrm{n}=5)$ cáncer digestivo, 2,7\% $(n=4)$ linfomas, 2\% ( $n=3)$ tumores de cabeza y cuello, $2 \%(n=3)$ sarcomas, $1.4 \%$ $(n=2)$ dermatológicos y $4.1 \quad(n=6)$ otros. El $27 \%(n=40)$ de los pacientes se encontraban en estadio I, 33,1\% $(n=49)$ en estadio II, $19,6 \% \quad(n=29)$ en el estadio III, $16.9 \%$ $(n=25)$ en el estadio IV, 3,4\% $(n=5)$ no estadio. El $85,1 \% \quad(n=126)$ se encontraban recibiendo tratamiento médico oncológico y $14,9 \%(n=22)$ no.

\section{Instrumentos de evaluación}

El Cuestionario de Afrontamiento al Estrés para Pacientes Oncológicos (CAEPO) de González ${ }^{(1)}$ consta de 40 ítems y 7 escalas con valores de consistencia interna altos según informa el autor. El rango de respuesta de los 40 ítems es de 4 puntos (de 0 "nunca" a 3 "casi siempre").

Siguiendo los resultados mostrados por el autor las escalas se denominan: Enfrentamiento y Lucha Activa (ELA) con 9 indicadores (ítems: 1, 2, 13, 16, 17, 21, 24, 31 y 39) $(\alpha=0,92)$; Autocontrol y Control Emocional (ACE) con 7 indicadores (ítems: $3,7,12,19,25,28$ y 40) ( $\alpha=0,90)$; Búsqueda de Apoyo Social (BAS) con 4 indicadores (ítems: 5, 10, 15 y 33) $(\alpha=0,89)$; Ansiedad y Preocupación Ansiosa (APA) con 6 indicadores (ítems: 4, 6, 14, 22, 26 y 32) $(\alpha=0,91$; Pasividad y Resignación Pasiva (PRP) con 5 indicadores (ítems: 11, 23, $34,35$ y 37$)(\alpha=0,88)$; Huida y Distanciamiento (HD) con 6 indicadores (ítems: 8, $9,18,29,30$ y 38) $(\alpha=0,91)$ y, por último, Negación (N) con 3 indicadores (ítems: 20, 27 y 36) $(\alpha=0,78)$.

También se indica que los primeros tres componentes (ELA, ACE y BAS) muestran 
la presencia de afrontamiento adaptativo ante la situación de enfermedad, caracterizado por pensamientos y conductas positivas y/o activas, mientras que los restantes cuatro componentes (APA, PRP, HD y N) se refieren al afrontamiento desadaptativo ante la situación de enfermedad, caracterizado por pensamientos y conductas negativas $y / o$ pasivas.

\section{Procedimiento}

A los pacientes que acuden a la Unidad de Psicología de la Fundación IVO se les propuso, entre la primera y segunda sesiones de evaluación, formar parte del estudio. Firmaron el consentimiento informado, garantizándose el anonimato y confidencialidad de la información de acuerdo con las normas éticas de investigación de la Declaración de Helsinki (Asociación Médica Mundial, 2008) y la Ley Orgánica 15/1999, de 13 de diciembre, de Protección de Datos de Carácter Personal. Los participantes respondieron por escrito el instrumento de medición en presencia de las psicólogas de la Unidad de Psicooncología.

El proyecto fue revisado en sus aspectos éticos y técnicos por el Comité Ético de Investigación Clínica (CEIC) de la Fundación IVO.

\section{ANÁLISIS ESTADÍSTICOS}

La consistencia interna se estimó por el coeficiente alfa de Cronbach $(\alpha)$. Se consideraron valores altos aquellos $\geq 0,70$, adecuados $\geq 0,60$ y bajos $<0,60$. El ajuste de la distribución a una curva normal se contrastó por la prueba de KolmogorovSmirnov (ZK-S). La estructura factorial se determinó tanto por análisis factorial exploratorio (AFE) por Componentes Principales con rotación varimax, así como por análisis factorial confirmatorio (AFC) por Máxima Verosimilitud (ML). El número de factores se determinó por el criterio Kaiser (autovalores mayores a 1), por la expectativa y por el criterio de Scree Test ${ }^{(25)}$. Se eliminaron los ítems con cargas factoriales menores que .40 en la matriz de estructura. En el AFC se manejaron siete índices de ajuste: tres básicos (función de discrepancia [FD], chi-cuadrado [ $\chi 2]$ y cociente entre chi-cuadrado y sus grados de libertad $\left.\left[\chi^{2 / g l}\right]\right)$; dos poblacionales de no centralidad (parámetro de no centralidad poblacional [PNCP] y residuo cuadrático medio de aproximación [RMSEA] de Steiger-Lind); además dos índices comparativos (índice de bondad de ajuste [GFI] de Jöreskog y Sörbom y su modalidad corregida [AGFI]). Se estipularon como valores de buen ajuste para los índices: $p$ de $\chi^{2}>$ 0,05 , FD y $\chi 2 / g \mid<2$, PNCP $<1$, RMSEA $<0,05$, GFI > 0,95 y AGFI > 0,90; y como valores adecuados: $p$ de $\chi^{2}>0,01$, FD y $\chi 2 / \mathrm{gl}<3$, PNCP $<2$, RMSEA $<0,08$, GFI $>0,85$ y AGFI $>0,80$. Los cálculos estadísticos se realizaron con SPSS22 y AMOS22.

\section{RESULTADOS}

\section{Análisis factorial exploratorio}

Aplicando el criterio de Kaiser (autovalores mayores a 1), el número de factores fue diez explicándose el $63,71 \%$ de la varianza total. Sin embargo, la solución de diez factores se descartó debido a que a partir del quinto factor el número de indicadores fueron insuficientes. El criterio de Scree Test planteó cuatro factores.

Al forzar la solución a 7 factores conforme a la propuesta original de González ${ }^{(1)}$, se explicó el $54,82 \%$ de la varianza total. Tras la rotación ortogonal por el método varimax, se definió un primer factor conformado por ocho ítems de ELA $(1,2,13,16,17$, 21,24 y 39), y 6 ítems de $\operatorname{ACE}(3,7,12,25$, 28 y 40), el valor de consistencia interna de los 14 ítems fue alto $(\alpha=0,90)$. El segundo factor quedó conformado por cinco ítems, 
dos correspondientes al factor original de APA (ítems: 14 y 22), uno correspondiente a BAS (ítem: 15), uno correspondiente a $\mathrm{N}$ (ítem: 36), y uno correspondiente a HD (ítem: 29), el valor de consistencia interna de los cinco ítems fue alto $(\alpha=0,76)$. El tercer factor quedó conformado por cuatro ítems, tres corresponden al factor original de BAS (ítems: 5, 10 y 33), y uno a ACE (ítem: 19), el valor de consistencia interna de los 4 ítems fue alto $(\alpha=0,78)$. El cuarto factor quedó conformado por los cinco ítems que conforman el factor original de PRP (ítems: 11, 23, 34, 35 y 37), el valor de consistencia interna fue alto $(\alpha=0,75)$. El quinto factor quedó conformado por cuatro ítems de HD (ítems: 8, 9, 18 y 38), el valor de consistencia interna fue adecuado $(\alpha=$ $0,64)$. El sexto factor quedó conformado por seis ítems, cuatro ítems de APA (ítems: 4, 6, 26 y 32) y dos ítems de $N$ (ítems: 20 y 27), el valor de consistencia interna fue adecuado $(\alpha=0,64)$. El séptimo factor quedó conformado por dos ítems, uno de HD (ítem: 30) y otro de ELA (ítem: 31), el valor de consistencia interna fue muy bajo $(\alpha=0,27)$. Por lo tanto, la solución de siete factores también se descartó debido a que el número de indicadores fue insuficiente en el último factor.

Al forzar la solución a cuatro factores conforme al criterio de Cattell ${ }^{(25)}$, se explicó el $43,47 \%$ de la varianza total. Tras la rotación ortogonal por el método varimax, se definió un primer factor conformado por 17 ítems, ocho ítems de ELA (ítems: 1, 2, 13, 16, 17, 21, 24 y 39), seis ítems de ACE (ítems: 3, 7, 12, 19, 28 y 40), y tres ítems de BAS (ítems: 5, 10 y 33). Por su contenido se le denominó Afrontamiento Activo $(\mathrm{AA})$, el valor de consistencia interna de los 17 ítems fue alto $(\alpha=0,90)$. El segundo factor está conformado por siete ítems, se agruparon cuatro ítems de APA (4, 14, 22 y 32), uno de BAS (ítem: 15), uno de $\mathrm{N}$ (ítem: 36) y uno de HD (ítem: 29). Por su contenido se le denominó Rumiación y Preocupación Ansiosa (RPA), el valor de consistencia interna de los 7 ítems fue alto $(\alpha=0,77)$. El tercer factor está conformado por siete ítems, se agruparon cuatro ítems de HD (ítems: 8, 9, 18 y 38), dos de $\mathrm{N}$ (ítems: 20 y 27), y uno de APA (ítem: 6). Por su contenido se le denominó Evitación y Negación (EN), el valor de consistencia interna de los 7 ítems fue adecuado $(\alpha=$ 0,68), al eliminar el ítem 6 de ansiedad y preocupación ansiosa la consistencia interna incrementa ligeramente $(\alpha=0,69)$. Se opta por eliminar el ítem 6 por poseer un contenido diferente con el resto de los ítems. El cuarto factor está conformado por los cinco ítems originales de PRP (ítems: $11,23,34,35$ y 37$)$. Por su contenido se le denominó Pasividad y resignación el valor de consistencia interna de los 5 ítems fue alto $(\alpha=0,75)$. Los ítems $25,26,30$ y 31 tuvieron cargas menores a 0,40 (véase tabla 1).

\section{Análisis factorial confirmatorio}

Se contrastó el modelo original de siete factores con 40 ítems propuesto por González ${ }^{(1)}$. Asimismo, se contrastó un modelo de cuatro factores correlacionados con 35 ítems, tal como sugiere el análisis factorial exploratorio.

El modelo original de siete factores con 40 ítems propuesto por González ${ }^{(1)}$ arrojó una solución inadecuada por lo que el programa no proporcionó sus parámetros. El modelo de cuatro factores correlacionados con 35 ítems mostró todos los parámetros significativos. De los índices de ajuste contemplados, uno reflejó buen ajuste $(\chi 2 / g)=$ 1,89 ), uno mostró un ajuste adecuado (RMS $E A=0,08)$, y cuatro mostraron un mal ajuste $(G F I=0,72, A G F I=0,68, N F I=0,56, C F I$ $=0,73)$. El ajuste del modelo se rechazó por la prueba chi-cuadrado $(\chi 2(556, N=148)=$ $1053,96, p<0,01)$. Las correlaciones fueron directas entre pasividad y resignación con evitación y negación $(r=0,47, p<$ 


\section{Tabla 1. Matriz estructural}

\begin{tabular}{|c|c|c|c|c|}
\hline \multirow{2}{*}{ Ítems } & \multicolumn{4}{|c|}{ Componentes } \\
\hline & 1 & 2 & 3 & 4 \\
\hline ELA 21 Procuro ser fuerte y plantar cara a mi problema de salud. & ,76 & & & \\
\hline ELA 24 Confío en mi fuerza de voluntad para que todo salga bien. &, 72 & & & \\
\hline ELA 13 Creo que esta situación pasará pronto y volveré cuanto antes a mi vida habitual. & ,72 & & & \\
\hline ACE 7 Pienso que, respecto a lo que me ocurre ahora, las cosas me van a salir bien. & ,72 & & & \\
\hline ELA 2 Estoy dispuesto a plantarle cara a esta situación, aunque esté preocupado o nervioso. & ,70 & & & \\
\hline ELA 39 Creo que si en otras ocasiones he superado mis problemas, ahora también lo haré. & ,69 & & & \\
\hline ACE 12 Trato de convencerme a mi mismo de que todo va a salir bien. & ,67 & & & \\
\hline ELA 17 Estoy dispuesto a luchar para que todo salga bien. & ,67 & & & \\
\hline ACE 3 Cuando estoy preocupado como ahora, procuro pensar en algo agradable y positivo. & ,66 & & & \\
\hline ACE 40 Puedo distraerme y no pensar en cosas negativas respecto a mi situación actual. & ,61 & & & \\
\hline ACE 19 Intento compartir mis emociones con algunas personas. &, 57 & & & \\
\hline BAS10 Hablo de mis preocupaciones con personas que me son cercanas: familia, amigos, etc. &, 56 & & & \\
\hline $\begin{array}{l}\text { ELA } 16 \text { Siento que lo que me está sucediendo me ayuda a saber de verdad las cosas que son } \\
\text { importantes en la vida. }\end{array}$ &, 55 & & & \\
\hline ACE 28 Intento mostrarme sereno y animado. &, 55 & & & \\
\hline BAS 5 He procurado hablar con aquellas personas que pueden ayudarme en mi situación. &, 52 & & & \\
\hline BAS 33 Muestro mis sentimientos y estados de ánimo ante las personas importantes para mí. &, 50 & & & \\
\hline $\begin{array}{l}\text { ELA } 1 \text { Habitualmente cuando me siento nervioso, procuro hacer cosas que me tranquilicen tales } \\
\text { como pasear, leer, ver la televisión u otras cosas. }\end{array}$ & ,40 & & & \\
\hline
\end{tabular}

ELA 31 Hago todo lo que me mandan porque creo que es bueno para mi recuperación.

ACE 25 Procuro controlar mis emociones.

BAS 15 Creo que voy a necesitar la ayuda de otras personas para superar esto.

N 36 La verdad es que estoy muy preocupado por mi salud.

APA 22 Siempre que estoy solo, pienso en lo que me está sucediendo ahora.

HD 29 Pienso constantemente lo que sería mi vida si no hubiera enfermado.

APA 14 Pienso constantemente en el problema que tengo con mi salud.

APA 4 Si estoy preocupado suelo pensar en cosas que me hacen sentir peor.

APA 32 Creo que aunque supere esta situación, quedaré muy afectado y nada será como antes.

, 46

APA 26 Pienso que no voy a ser capaz de soportar esta situación.

HD 9 Evito hablar de lo que me pasa porque no me gusta este tema.

N 20 Creo que los demás exageran y que en realidad lo que me sucede carece de importancia.

N 27 Pienso que pueden haberse equivocado respecto a la gravedad de mi problema de salud.

HD 38 Procuro evadirme y no pensar en lo que me pasa.

APA 6 Pienso que esto que me sucede es porque no me he ocupado adecuadamente de mi salud.

HD 8 Prefiero olvidarme de lo que me ocurre y no pensar en ello.

HD 18 Me gusta pensar que lo que me sucede no es real, que no me pasa nada.

PRP 35 Pienso que las cosas ocurren y que nada puede hacer para cambiar los acontecimientos.

PRP 23 Pienso que lo mejor es dejar que las cosas sigan su curso.

PRP 11 Pienso que en este caso no puedo hacer nada y lo mejor es aceptar las cosas como son.

PRP 37 Creo que en esta situación lo mejor es aceptar las cosas como vienen.

PRP 34 Me doy por vencido en esta situación y pienso que "lo que tenga que ser será".

HD 30 Creo que la mejor solución para que todo salga bien es rezar.

Método de extracción: componentes principales. Método de rotación: Varimax. La rotación convergió en 6 iteraciones. 
0,01), y con rumiación y preocupación ansiosa $(r=0,35, p<0,01)$; e inversas entre afrontamiento activo y rumiación y preocupación ansiosa $(r=-0,30, p<0,01)$.

Tras revisar los índices de modificación $(\mathrm{MI}=43,04)$ se introdujo una correlación entre los residuos de los ítems 10 y 19 que hacen referencia a la expresión emocional. Al especificar esta correlación entre estos dos residuos $(r=0,54, p<0,01)$ se logró mejorar ligeramente el modelo. El ajuste del modelo se rechazó por la prueba chicuadrado $(\chi 2(556, N=148)=1004,10, p<$ $0,01)$. Todos los parámetros del modelo fueron significativos. De los índices de ajuste contemplados, uno reflejó buen ajuste $(\chi 2)$ $g l=1,81)$, uno mostró un ajuste adecuado
(RMS $E A=0,07)$, y cuatro mostraron un mal ajuste $(G F I=0,73, A G F I=0,70, N F I=$ $0,58$ y $C F I=0,75)$ (véase la figura 1 ).

Distribución de los Factores Finales del Cuestionario

Tras los resultados del análisis factorial exploratorio y confirmatorio se reduce la escala a 35 ítems, con cuatro estrategias de afrontamiento (rumiación y preocupación ansiosa, evitación y negación, pasividad y resignación, y afrontamiento activo). Los factores de afrontamiento activo, rumiación y preocupación ansiosa, y pasividad y resignación mostraron valores de consistencia interna altos y una distribución que

Figura 1. Modelo estandarizado de cuatro factores con 35 ítems estimado por ML

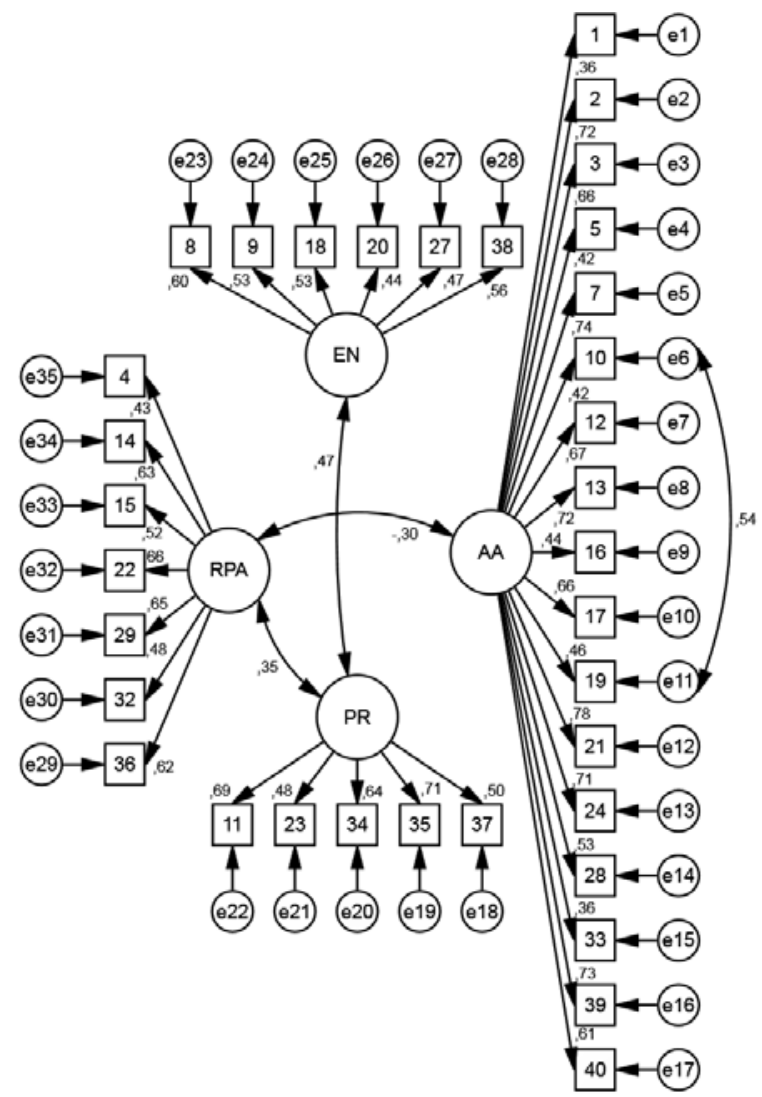


se ajustó a una curva normal. El factor de evitación y preocupación ansiosa mostró una consistencia interna adecuada y no se ajustó a una curva normal $\left(Z_{K-S}=1,67, p=\right.$ 0,01) (véase la tabla 2).

$\mathrm{Al}$ analizar las diferencias de medias de las cuatro estrategias de afrontamiento entre mujeres y hombres, no se encontraron diferencias significativas (véase la tabla 3).

\section{DISCUSIÓN}

Se esperaba una estructura de siete factores con los 40 ítems que conforman el CAEPO siguiendo la propuesta original de González ${ }^{(1)}$; no obstante, los resultados del análisis factorial exploratorio y confirmatorio, arrojaron una estructura de cuatro factores correlacionados con 35 ítems. De los 40 ítems originales, cinco fueron eliminados, cuatro ítems por presentar un peso factorial menor a 0,40 en el análisis factorial exploratorio (ítems: 25, 26, 29 y 30) y uno (ítem: 6) por mejorar la consistencia interna del factor de evitación y negación al ser eliminado. En pacientes no oncológicos, Orive et al. ${ }^{(22)}$ también encontraron una estructura de cuatro factores; sin embargo, dicha estructura difiere con la encontrada en la presente muestra de pacientes oncológicos.

En el presente estudio, el primer factor lo re-etiquetamos como afrontamiento activo debido a que agrupó los factores originales de enfrentamiento y lucha activa, autocontrol y control emocional, y búsqueda de

Tabla 2. Consistencia, descriptivos y ajuste a la normalidad ( $\mathbf{N}=\mathbf{1 4 8}$ )

\begin{tabular}{cccccccccccc}
\hline & & \multicolumn{1}{c}{ Descriptivos de la distribución } & \multicolumn{4}{c}{ Normalidad } \\
\cline { 3 - 10 } CAEPO-35 & $\alpha$ & $M$ & Mdn & DE & Min & Max & $S$ & $C$ & $Z_{K-S}$ & $p$ \\
\hline AA &, 90 & 33,26 & 34 & 9,81 & 4 & 51 &,- 28 &,- 49 &, 90 &, 39 \\
RPA &, 77 & 10,95 & 11 & 4,50 & 1 & 21 &,- 08 &,- 59 & 1,07 &, 21 \\
EN &, 69 & 5,66 & 5 & 3,61 & 0 & 16 &, 68 &,- 19 & 1,67 &, 01 \\
PR &, 75 & 7,26 & 7 & 3,69 & 0 & 15 &, 19 &,- 62 & 1,29 &, 07 \\
\hline
\end{tabular}

$\mathrm{AA}=$ afrontamiento activo, $\mathrm{RPA}=$ rumiación y preocupación ansiosa, $\mathrm{EN}=$ evitación y negación, $\mathrm{PR}=$ pasividad y resignación. $S=$ sesgo o asimetría y $C=$ curtosis. $E T$ de $S=0,20$ y $E T$ de $C=0,40$.

\section{Tabla 3. Comparación de medias entre mujeres y hombres}

\begin{tabular}{|c|c|c|c|c|c|c|c|c|c|c|c|}
\hline \multirow{2}{*}{ CAEPO-35 } & \multirow{2}{*}{ Sexo } & \multicolumn{3}{|c|}{ Descriptivos } & \multicolumn{2}{|c|}{ Levene } & \multicolumn{3}{|c|}{ Student } & \multicolumn{2}{|c|}{ Diferencia } \\
\hline & & $N$ & M & $D E$ & $F$ & $p$ & $t$ & $g l$ & $p$ & $D M$ & $E E$ \\
\hline \multirow{2}{*}{ AA } & M & 122 & 33,49 & 9,59 & \multirow{2}{*}{,08 } & \multirow{2}{*}{,78 } & \multirow{2}{*}{,63 } & \multirow{2}{*}{146} & \multirow{2}{*}{,53 } & \multirow{2}{*}{1,34} & \multirow{2}{*}{2,12} \\
\hline & $\mathrm{H}$ & 26 & 32,15 & 10,91 & & & & & & & \\
\hline \multirow[b]{2}{*}{ RPA } & M & 122 & 11,16 & 4,42 & \multirow{2}{*}{,11 } & \multirow{2}{*}{,74 } & \multirow{2}{*}{1,24} & \multirow{2}{*}{146} & \multirow{2}{*}{,22 } & \multirow{2}{*}{1,20} & \multirow{2}{*}{, 97} \\
\hline & $\mathrm{H}$ & 26 & 9,96 & 4,83 & & & & & & & \\
\hline \multirow[b]{2}{*}{ EN } & M & 122 & 5,54 & 3,70 & \multirow{2}{*}{1,81} & \multirow{2}{*}{, 18 } & \multirow{2}{*}{,- 89} & \multirow{2}{*}{146} & \multirow{2}{*}{,38 } & \multirow{2}{*}{,- 69} & \multirow{2}{*}{, 78} \\
\hline & $\mathrm{H}$ & 26 & 6,23 & 3,12 & & & & & & & \\
\hline \multirow{2}{*}{ PR } & M & 122 & 7,34 & 3,82 & \multirow{2}{*}{3,47} & \multirow{2}{*}{, 06} & \multirow{2}{*}{,62 } & \multirow{2}{*}{146} & \multirow{2}{*}{, 53} & \multirow{2}{*}{, 50} & \multirow{2}{*}{,80 } \\
\hline & $\mathrm{H}$ & 26 & 6.85 & 3.08 & & & & & & & \\
\hline
\end{tabular}

$\overline{\mathrm{AA}}=$ afrontamiento activo, $\mathrm{RPA}=$ rumiación y preocupación ansiosa, $\mathrm{EN}=$ evitación y negación, $\mathrm{PR}=$ pasividad y resignación. 
apoyo social. Este primer factor corresponde al factor original de segundo orden denominado Afrontamiento Positivo por Gonzá$l e z^{(1)}$. Este tipo de afrontamiento favorece la adaptación a la situación de enfermedad, debido a que el paciente afronta la situación manejando sus estados emocionales negativos, se muestra colaborador y con apertura para recibir apoyo emocional ${ }^{(1)}$.

El segundo factor lo re-etiquetamos como rumiación y preocupación ansiosa debido a que agrupó ítems que hacen referencia a pensamientos repetitivos, los cuales solo giran en torno a los acontecimientos del pasado, y en los síntomas y sentimientos que estos producen ${ }^{(26)}$, así como en las causas y consecuencias de la situación adversa que se está viviendo, sin progresar de forma activa en la solución ${ }^{(27)}$. En este sentido, en el AFC se encontró que a mayor rumiación y preocupación ansiosa menor afrontamiento activo. Es decir, que el preocuparse y tener pensamientos repetitivos y negativos no permite que la persona adopte comportamientos efectivos que favorezcan el afrontar activamente el proceso del cáncer. Cabe señalar, que en la versión original de González ${ }^{(1)}$ el ítem 36 formaba parte del factor de negación y se sumaba de manera inversa. En la versión reducida del CAEPO que proponemos en este estudio, el ítem 36: "la verdad es que estoy muy preocupado por mi salud" forma parte del factor de rumiación y preocupación ansiosa, y se suma de manera directa.

El tercer factor lo re-etiquetamos como evitación y negación debido a que agrupó ítems que hacen referencia al hecho de evitar pensamientos sobre la situación por la que se está atravesando. Un gran número de autores ya habían asociado anteriormente estos dos constructos, refiriéndose a ellos como la necesidad de distanciarse, evitar y suprimir pensamientos y emociones $^{(28-31)}$. Según, Rabinowitz y Peirson ${ }^{(32)}$ al negar la situación, la persona se defiende de pensamientos, sentimientos o de infor- mación dolorosa o amenazante; manifestándose tanto en personas sanas como en personas con alguna enfermedad, y/o en los familiares del paciente.

El cuarto factor fue el único que agrupó los cinco ítems originales de pasividad y resignación de González ${ }^{(1)}$. En el AFC, el factor de pasividad y resignación se asoció positivamente con evitación y negación, y con rumiación y preocupación ansiosa. Ambas relaciones ponen de manifiesto que el no hacer nada y el resignarse solo conduce a que la persona se encuentre continuamente evitando y generando pensamientos negativos que producen que la situación se experimente con mayor malestar emocional|(33).

Los valores de consistencia interna fueron altos para afrontamiento activo, rumiación y preocupación ansiosa, y pasividad y resignación. El factor de evitación y negación obtuvo valores de consistencia interna adecuada. En otros estudios, utilizando la estructura original de González ${ }^{(1)}$, los valores de consistencia interna para los factores de huida y distanciamiento, y negación fueron bajos $^{(20-22)}$. Sin embargo, en el presente estudio, al agrupar ambos factores, se mejora considerablemente la consistencia interna. La distribución de los datos se ajustó a una curva normal en tres de los cuatro factores: afrontamiento activo, rumiación y preocupación ansiosa, y pasividad y resignación.

$\mathrm{Al}$ analizar las diferencias de medias de las cuatro estrategias de afrontamiento entre mujeres y hombres, no se encontraron diferencias significativas. Asimismo, en diversos estudios se ha encontrado que tanto los hombres como las mujeres con cáncer afrontan de manera similar el proceso del cáncer ${ }^{(21,34)}$.

\section{LIMITACIONES}

Como limitaciones del estudio debe señalarse el carecer de un muestreo probabilístico, habiéndose realizado en una 
muestra incidental de pacientes que acudían a la Unidad de Psicología de la Fundación IVO, por lo que los resultados no deben manejarse como generalizaciones. Todos los datos son de autoinforme, por lo que pueden diferir de otros procedentes de entrevistas, observaciones, pruebas proyectivas o tiempos de reacción.

Otra de las limitaciones es la diferencia del tamaño de muestra entre hombres y mujeres. Inicialmente se pretendía obtener una muestra equivalente de al menos 200 participantes, la mitad mujeres y la mitad hombres. Sin embargo, se encontró que las mujeres acuden con mayor frecuencia a la Unidad de Psicología de la Fundación IVO. Se sugiere que en futuras investigaciones se obtenga una muestra equivalente de sexos, para poder contrastar la estructura factorial exploratoria y confirmatoria del CAEPO.

\section{CONCLUSIONES}

En conclusión se aconseja reducir la escala CAEPO a 35 ítems (véase Anexo 1), utilizando todos los ítems de manera directa; es decir que a mayor puntuación, mayor es la frecuencia en la que experimentan cada una de las estrategias de afrontamiento. El CAEPO presentó una estructura de cuatro factores, y se propone una nueva nominación más adecuada al contenido de los mismos: 1) afrontamiento activo, 2) rumiacion y preocupación ansiosa, 3) evitación y negación, y 4) pasividad y resignación. El modelo de cuatro factores correlacionados tuvo un ajuste a los datos de bueno a malo. El modelo del análisis factorial confirmatorio se sostiene por los valores de consistencia interna e interpretación de los factores. Los valores de consistencia interna para los cuatro factores fueron altos, salvo para el factor de evitación y negación el cual obtuvo una consistencia interna adecuada. La distribución de los cuatro factores se ajustaron a una curva normal, sólo el factor de evitación y negación se alejó de la norma- lidad. La manera de afrontar el proceso de enfermedad fue estadísticamente equivalente entre hombres y mujeres.

Así, los resultados arrojan que el CAEPO con 35 ítems y cuatro factores correlacionados es una escala consistente y válida.

\section{REFERENCIAS BIBLIOGRÁFICAS}

1. González MT. Cuestionario de afrontamiento al estrés para pacientes oncológicos. Madrid: TEA Ediciones, 2004.

2. Kazak AE. Evidence based interventions for survivors of childhood cancer and their families. J Pediatric Psychol 2005; 30:29-40.

3. Fernández Al. Alteraciones psicológicas asociadas a los cambios en la apariencia física en pacientes oncológicos. Psicooncología 2004;1:169-80.

4. López JG, Chimal AM, Valdés JM., Nava PB, Peña GMG. Acontecimientos productores de estrés en pacientes oncológicos. Psicol Iberoamericana 2012;1:46-57.

5. Jaman-Mewes P, Rivera MS. Vivir con cáncer: una experiencia de cambios profundos provocados por la quimioterapia. Aquichan 2014;14:20-31.

6. Bragado C, Fernández A. Tratamiento psicológico del dolor y la ansiedad evocados por procedimientos médicos invasivos en oncología pediátrica. Psicothema 1996;8:625-56.

7. Lazarus RS. Stress and emotion. A new synthesis. New York: Springer, 1999.

8. Sandín B. El estrés: un análisis basado en el papel de los factores sociales. Intern J Clin Health Psychol 2003;3:141-57.

9. Mera PC, Ortiz M. La relación del optimismo y las estrategias de afrontamiento con la calidad de vida de mujeres con cáncer de mama. Ter Psicol 2012;30:69-78. Doi: 10.4067/S0718-48082012000300007.

10. Reich M, Remor, E. Variables psicosociales asociadas con calidad de vida relacionada con la salud en mujeres con cáncer de mama post-cirugía: una revisión sistemática. Ciencias Psicológicas 2010;4:177-221. 
11. Cardenal V, Cerezo MV, Martínez J, OrtizTallo M, Blanca MJ. Personality, emotions and coping styles: predictive value for the evolution of cancer patients. Spanish J Psychol 2012;15:756-67. Doi: 10.5209/ rev_SJOP.2012.v15.n2.38887.

12. Lazarus RS, Folkman S. Stress, appraisal and coping. New York: Springer Publishing, 1984

13. Sandín B, Chorot P. Cuestionario de afrontamiento del estrés (CAE): desarrollo y validación preliminar. Rev Psicopatol Psicol Clin 2003;8:39-54.

14. Moos R. Coping Responses Inventory Psychological Assessment Resources. Inc. Florida. USA, 1993.

15. Watson $M$, Greer J, Inayat $Y$, Burgess $C$, Robertson B. Development of a questionnaire of adjustment to cancer: the MAC scale. Psychol Med 1988:18:203-9.

16. Watson M, Law M, Dos Santos M, Greer S, Baruch J, Bliss J. The Mini-MAC Further development of the Mental Adjustment to Cancer scale. J Psychos Oncol 1994;12:3346.doi:10.1300/J077V12N03_03

17. Cano A. Control emocional, estilo represivo de afrontamiento y cáncer: ansiedad y cáncer. Psicooncología 2005;2:71-80.

18. Font A, Cardoso A. Afrontamiento en cáncer de mama: pensamientos, conductas y reacciones emocionales. Psicooncología 2009;6:27-42.

19. González MT. Análisis de un instrumento de evaluación del afrontamiento del estrés en sujetos con cáncer. RIDEP 2008;1:35-49.

20. Díaz G, Yaringaño J. Clima familiar y afrontamiento al estrés en pacientes oncológicos. Rev Invest Psicol 2010;13: 69-86.

21. García-Conde A. Dimensiones básicas de la personalidad, afrontamiento y adaptación en pacientes oncológicos. Tesis doctoral: Universidad de Valencia, 2014.

22. Orive M, Quintana JM, Vrotsou K, Las Hayas C, Bilbao A, Barrio l, et al. Applying a coping with stress questionnaire for cancer patients to patients with non-cancer chronic illnesses. J Health Psychol 2013;18:73749. Doi:10.1177/1359105312464673.
23. Folkman S. The Oxford handbook of stress, health, and coping. New York: Oxford University Press, 2011.

24. Vargas J, Cervantes M, Aguilar J. Estrategias de afrontamiento del sida en pacientes diagnosticados como seropositivos. Psicol Salud 2009;19:215-21.

25. Cattell R. The Scree Test for the numbers of factors. Multivariate Behav Res 1966:1:141-61.

26. Bayés R. Tiempo y duelo. Med Paliat 2008; 15:194-5.

27. Nolen-Hoeksema S. Responses to depression and their effects on the duration of depressive episodes. J Abnormal Psychol 1991;100:569-82. Doi:10.1037//0021843X.100.4.569.

28. Levine J, Rudy T, Kerns R. A two factor model of denial of illness. A confirmatory factor analysis. J Psychosom Res 1994;38:99-110.

29. Moorey S, Greer S. Psychological therapy for patients with cancer: a new approach. Londres: Heineman Medical Books, 1989.

30. Moyer A, Levine E. Clarification of the conceptualization and measurement of denial in psychosocial oncology research. Ann Behav Med 1998;20:149-60.

31. Wheeler S, Lord L Denial: A conceptual analysis. Arch Psychiatr Nurs 1999;13:31120. Doi:10.1016/S0883-9417(99)80063-6

32. Rabinowitz T, Peirson R. "Nothing is wrong, Doctor": Understanding and managing denial in patients with cancer. Cancer Invest 2016;24:68-71. Doi: 10.1080/07357900500449678

33. Behen JM, Rodrigue JR. Predictors of coping strategies among adults with cancer. Psychological Rep 1994;74:43-8. Doi: 10.2466/pr0.1994.74.1.43.

34. McCaughan E, Prue G, Parahoo K, Mcllfatrick S, McKenna $H$. Exploring and comparing the experience and coping behaviour of men and women with colorectal cancer after chemotherapy treatment: A qualitative longitudinal study. Psychooncology 2012;21:64-71. Doi:10.1002/pon.1871. 


\section{Anexo 1 \\ CAEPO: versión original con 40 ítems y versión reducida con 35 ítems}

A continuación aparecen una serie de frases que se refieren a distintas formas de actuar ante situaciones difíciles o problemáticas. La situación en la que usted se encuentra ahora podría considerarse que tiene estas características. Indique, rodeando con un círculo, con cual de dichas formas se identifica más su manera de COMPORTARSE EN ESTA SITUACIÓN. No hay respuestas buenas ni malas ya que cada persona puede resolver sus problemas de la forma que considera más conveniente. Procure contestar con la mayor sinceridad.

Hay cuatro opciones posibles en cada cuestión:

\begin{tabular}{|c|c|c|c|}
\hline 0 & 1 & 2 & 3 \\
\hline Nunca & Algunas veces & Muchas veces & Casi siempre \\
\hline
\end{tabular}

\begin{tabular}{|c|c|c|c|c|c|c|}
\hline O & $R$ & Ítems & & & & \\
\hline 1 & 1 & $\begin{array}{l}\text { Habitualmente cuando me siento nervioso, procuro hacer cosas } \\
\text { que me tranquilicen tales como pasear, leer, ver la televisión u otras } \\
\text { cosas. }\end{array}$ & 0 & 1 & 2 & 3 \\
\hline 2 & 2 & $\begin{array}{l}\text { Estoy dispuesto a plantarle cara a esta situación, aunque esté } \\
\text { preocupado o nervioso. }\end{array}$ & 0 & 1 & 2 & 3 \\
\hline 3 & 3 & $\begin{array}{l}\text { Cuando estoy preocupado como ahora, procuro pensar en algo } \\
\text { agradable y positivo. }\end{array}$ & 0 & 1 & 2 & 3 \\
\hline 4 & 4 & Si estoy preocupado suelo pensar en cosas que me hacen sentir peor. & 0 & 1 & 2 & 3 \\
\hline 5 & 5 & $\begin{array}{l}\text { He procurado hablar con aquellas personas que pueden ayudarme } \\
\text { en mi situación. }\end{array}$ & 0 & 1 & 2 & 3 \\
\hline 6 & $\mathrm{E}$ & $\begin{array}{l}\text { Pienso que esto que me sucede es porque no me he ocupado } \\
\text { adecuadamente de mi salud. }\end{array}$ & 0 & 1 & 2 & 3 \\
\hline 7 & 6 & $\begin{array}{l}\text { Pienso que, respecto a lo que me ocurre ahora, las cosas me van a } \\
\text { salir bien. }\end{array}$ & 0 & 1 & 2 & 3 \\
\hline 8 & 7 & Prefiero olvidarme de lo que me ocurre y no pensar en ello. & 0 & 1 & 2 & 3 \\
\hline 9 & 8 & Evito hablar de lo que me pasa porque no me gusta este tema. & 0 & 1 & 2 & 3 \\
\hline 10 & 9 & $\begin{array}{l}\text { Hablo de mis preocupaciones con personas que me son cercanas: } \\
\text { familia, amigos, etc. }\end{array}$ & 0 & 1 & 2 & 3 \\
\hline 11 & 10 & $\begin{array}{l}\text { Pienso que en este caso no puedo hacer nada y lo mejor es aceptar } \\
\text { las cosas como son. }\end{array}$ & 0 & 1 & 2 & 3 \\
\hline 12 & 11 & Trato de convencerme a mi mismo de que todo va a salir bien. & 0 & 1 & 2 & 3 \\
\hline 13 & 12 & $\begin{array}{l}\text { Creo que esta situación pasará pronto y volveré cuanto antes a mi } \\
\text { vida habitual. }\end{array}$ & 0 & 1 & 2 & 3 \\
\hline 14 & 13 & Pienso constantemente en el problema que tengo con mi salud. & 0 & 1 & 2 & 3 \\
\hline 15 & 14 & $\begin{array}{l}\text { Creo que voy a necesitar la ayuda de otras personas para superar } \\
\text { esto. }\end{array}$ & 0 & 1 & 2 & 3 \\
\hline
\end{tabular}




\begin{tabular}{|c|c|c|c|c|c|c|}
\hline 16 & 15 & $\begin{array}{l}\text { Siento que lo que me está sucediendo me ayuda a saber de verdad } \\
\text { las cosas que son importantes en la vida. }\end{array}$ & 0 & 1 & 2 & 3 \\
\hline 17 & 16 & Estoy dispuesto a luchar para que todo salga bien. & 0 & 1 & 2 & 3 \\
\hline 18 & 17 & $\begin{array}{l}\text { Me gusta pensar que lo que me sucede no es real, que no me pasa } \\
\text { nada. }\end{array}$ & 0 & 1 & 2 & 3 \\
\hline 19 & 18 & Intento compartir mis emociones con algunas personas. & 0 & 1 & 2 & 3 \\
\hline 20 & 19 & $\begin{array}{l}\text { Creo que los demás exageran y que en realidad lo que me sucede } \\
\text { carece de importancia. }\end{array}$ & 0 & 1 & 2 & 3 \\
\hline 21 & 20 & Procuro ser fuerte y plantar cara a mi problema de salud. & 0 & 1 & 2 & 3 \\
\hline 22 & 21 & Siempre que estoy solo, pienso en lo que me está sucediendo ahora. & 0 & 1 & 2 & 3 \\
\hline 23 & 22 & Pienso que lo mejor es dejar que las cosas sigan su curso. & 0 & 1 & 2 & 3 \\
\hline 24 & 23 & Confío en mi fuerza de voluntad para que todo salga bien. & 0 & 1 & 2 & 3 \\
\hline 25 & $\mathrm{E}$ & Procuro controlar mis emociones. & 0 & 1 & 2 & 3 \\
\hline 26 & $\mathrm{E}$ & Pienso que no voy a ser capaz de soportar esta situación. & 0 & 1 & 2 & 3 \\
\hline 27 & 24 & $\begin{array}{l}\text { Pienso que pueden haberse equivocado respecto a la gravedad de } \\
\text { mi problema de salud. }\end{array}$ & 0 & 1 & 2 & 3 \\
\hline 28 & 25 & Intento mostrarme sereno y animado. & 0 & 1 & 2 & 3 \\
\hline 29 & 26 & Pienso constantemente lo que sería mi vida si no hubiera enfermado. & 0 & 1 & 2 & 3 \\
\hline 30 & $\mathrm{E}$ & Creo que la mejor solución para que todo salga bien es rezar. & 0 & 1 & 2 & 3 \\
\hline 31 & $\mathrm{E}$ & $\begin{array}{l}\text { Hago todo lo que me mandan porque creo que es bueno para mi } \\
\text { recuperación. }\end{array}$ & 0 & 1 & 2 & 3 \\
\hline 32 & 27 & $\begin{array}{l}\text { Creo que aunque supere esta situación, quedaré muy afectado y } \\
\text { nada será como antes. }\end{array}$ & 0 & 1 & 2 & 3 \\
\hline 33 & 28 & $\begin{array}{l}\text { Muestro mis sentimientos y estados de ánimo ante las personas } \\
\text { importantes para mí. }\end{array}$ & 0 & 1 & 2 & 3 \\
\hline 34 & 29 & $\begin{array}{l}\text { Me doy por vencido en esta situación y pienso que "lo que tenga } \\
\text { que ser será". }\end{array}$ & 0 & 1 & 2 & 3 \\
\hline 35 & 30 & $\begin{array}{l}\text { Pienso que las cosas ocurren y que nada puede hacer para cambiar } \\
\text { los acontecimientos. }\end{array}$ & 0 & 1 & 2 & 3 \\
\hline 36 & 31 & La verdad es que estoy muy preocupado por mi salud. & 0 & 1 & 2 & 3 \\
\hline 37 & 32 & $\begin{array}{l}\text { Creo que en esta situación lo mejor es aceptar las cosas como } \\
\text { vienen. }\end{array}$ & 0 & 1 & 2 & 3 \\
\hline 38 & 33 & Procuro evadirme y no pensar en lo que me pasa. & 0 & 1 & 2 & 3 \\
\hline 39 & 34 & $\begin{array}{l}\text { Creo que si en otras ocasiones he superado mis problemas, ahora } \\
\text { también lo haré. }\end{array}$ & 0 & 1 & 2 & 3 \\
\hline 40 & 35 & $\begin{array}{l}\text { Puedo distraerme y no pensar en cosas negativas respecto a mi } \\
\text { situación actual. }\end{array}$ & 0 & 1 & 2 & 3 \\
\hline
\end{tabular}

$\mathrm{O}$ = numeración de los ítems originales de González (2004)(1) con 40 ítems. $\mathrm{R}$ = nueva numeración de los ítems que conforman la versión reducida con 35 ítems. E = ítems eliminados en la versión reducida. 